\title{
Current laboratory requirements for adrenocorticotropic hormone and renin/aldosterone sample handling are unnecessarily restrictive
}

\author{
Authors: Ali ] Chakera, ${ }^{\mathrm{A}}$ Timothy J McDonald, ${ }^{\mathrm{B}}$ Bridget A Knight, ${ }^{\mathrm{C}}$ Bijay Vaidya ${ }^{\mathrm{D}}$ and Angus $\mathrm{G}$ Jones ${ }^{\mathrm{E}}$
}

\begin{abstract}
Samples for adrenocorticotropic hormone (ACTH) and aldosterone/renin analysis usually require rapid transport to the receiving laboratory for immediate separation and freezing. In practice, this means assessment is limited to hospital settings and many samples are rejected. We examined whether these requirements are necessary by assessing the stability of $\mathrm{ACTH}$, aldosterone and renin over 48 hours in whole blood collected in serum gel and EDTA plasma from 31 participants. Our results show that ACTH collected into EDTA plasma is stable at room temperature for at least 6 hours, mean change at 6 hours $-2.6 \%$ ( $95 \%$ CI -9.7 to 4.5$)$. Both aldosterone and renin were stable collected on serum gel at room temperature for at least 6 hours: mean change aldosterone $+0.2 \%$ ( $95 \% \mathrm{CI}-3.6$ to 4.0$)$, renin $-1.9 \%$ (95\% CI -7.0 to 3.2$)$. Therefore, by using appropriate preservatives, ACTH and aldosterone/renin can be measured on samples collected at room temperature and processed within 6 hours. This would facilitate outpatient and emergency room assessment of these analytes.
\end{abstract}

KEYWORDS: ACTH, aldosterone, hormone stability, renin

\section{Introduction}

Adrenocorticotropic hormone (ACTH), aldosterone and renin are commonly measured in clinical practice in the investigation of hypertension (aldosterone and renin), adrenal insufficiency (ACTH, aldosterone and renin) and endogenous Cushing's syndrome $(\mathrm{ACTH}) .{ }^{1,2}$ When assessing these hormones

\footnotetext{
Authors: ${ }^{\text {A }}$ consultant physician, Brighton and Sussex University Hospitals NHS Trust, Brighton, UK; ${ }^{B}$ consultant clinical scientist and honorary senior lecturer, Royal Devon and Exeter NHS Foundation Trust, Exeter, UK and University of Exeter Medical School, Exeter, UK; ${ }^{C}$ senior research midwife, NIHR Exeter Clinical Research Facility, University of Exeter Medical School, Exeter, UK; ${ }^{D}$ consultant physician and honorary clinical professor, Royal Devon and Exeter NHS Foundation Trust and University of Exeter Medical School, Exeter, UK; ENIHR clinician scientist and honorary consultant physician, University of Exeter Medical School and Royal Devon and Exeter NHS Foundation Trust, Exeter, UK
}

laboratories commonly require rapid transport to the receiving laboratory for immediate separation and freezing; however, there is marked variation in sample handling requirements. ${ }^{3}$ At the time of writing, of the 17 UK providers of ACTH measurement, 12 recommend rapid sample processing (from immediately to within 1 hour), with two recommending samples are collected 'on ice. ${ }^{3}$ For measurement of renin, seven of 13 providers specify immediate sample processing. ${ }^{3}$ As well as variation in the acceptable time to sample separation and freezing, there is variation of the recommended specimen type, including EDTA plasma, serum gel and lithium-heparin tubes for all three assays. ${ }^{3}$ These restrictions limit the use of these tests to hospital settings and impose a burden on clinic staff who will often have to leave the clinic to take a sample to the hospital laboratory. When adrenal insufficiency is identified, an ACTH measurement before hydrocortisone therapy assists differentiation of primary hypoadrenalism (raised ACTH), from secondary (low ACTH); therefore, most protocols recommend sending a baseline Synacthen ${ }^{\mathrm{TM}}$ test sample for subsequent ACTH measurement if hypoadrenalism is confirmed. It can be difficult to diagnose the underlying cause if the baseline blood sample has not been transported appropriately.

These strict requirements may not apply, as recently demonstrated with C-peptide and insulin. ${ }^{4}$ We aimed to determine the stability of ACTH, aldosterone and renin (concentration) in whole blood collected into EDTA and serum containers at room and refrigerator temperature.

\section{Methods}

\section{Participants}

$50 \mathrm{~mL}$ of blood was collected from each of the 31 participants (15 ACTH, 16 aldosterone/renin) before 10 am after $\mathrm{a} \geq 8$ hour fast and following written informed consent. Samples were collected in the sitting position. To ensure a range of ACTH values, three participants in this group had primary hypoadrenalism and one had Nelson's syndrome, with samples taken prior to morning hydrocortisone. The remaining participants (11/15) were from an unselected cohort and were not known to have endocrine disease. Four aldosterone/ renin participants had treated hypertension. Assayed samples 
were donations to the Peninsula Research Bank as part of the EXTEND study, which received ethical approval from the North Somerset and South Bristol Research Ethics Committee (UK).

\section{Sample handling}

Blood was divided immediately into nine EDTA plasma and nine serum gel (Sarstedt, Leicester, UK) tubes. One EDTA plasma and serum gel were centrifuged immediately $(3,000 \mathrm{~g}$ for 10 minutes) and plasma/serum frozen at $-80^{\circ} \mathrm{C}$ for batched analysis (baseline sample). Of the remaining samples, half of each type were stored at room temperature $\left(20^{\circ} \mathrm{C}\right)$ and half refrigerated $\left(4^{\circ} \mathrm{C}\right)$. At $1,6,24$ and 48 hours post blood draw, one of each of the four samples (room and fridge EDTA plasma and room and fridge serum gel) was centrifuged and serum/plasma stored at $-80^{\circ} \mathrm{C}$ for batched analysis.

\section{Sample analysis}

ACTH was analysed on the Roche E170 immunoassay platform (Roche Diagnostics, Mannheim, Germany) by the blood sciences department of the Royal Devon and Exeter Hospital, UK. Aldosterone and renin were analysed using Siemens Coat-ACount solid phase radioimmunoassay (aldosterone) and Cisbio Bioassays immunoradiometric assay (renin) by the clinical chemistry department of the University Hospital Southampton. Assay performance is reported in Table 1 .

\section{Statistical analysis}

Percentage change from baseline (mean and 95\% CI) was calculated for all storage conditions, sample types and time points. EDTA plasma samples were compared with EDTA plasma baseline, and serum gel with serum gel baseline. Percentage changes of more than $10 \%$ from baseline concentration were considered clinically significant. Hormone levels in serum and plasma were compared using the Wilcoxon signed-rank test. Analysis was performed in Excel 2010 (Microsoft) and Stata Statistical Software: Release 13 (StataCorp).

\section{Results}

\section{Participant characteristics and baseline hormone levels}

Participant characteristics and baseline hormone levels are shown in Table 2 . Renin concentration was $14 \%$ lower at baseline in EDTA plasma than serum gel $(\mathrm{p}=0.04)$. Aldosterone and ACTH levels did not differ by sample medium.

\begin{tabular}{llllll} 
Table 1. Assay performance & & & \\
\multicolumn{7}{c}{$\begin{array}{l}\text { Lower } \\
\text { limit of } \\
\text { detection }\end{array}$} & $\begin{array}{l}\text { Upper } \\
\text { limit of } \\
\text { detection }\end{array}$ & $\begin{array}{l}\text { CV - } \\
\text { low }\end{array}$ & $\begin{array}{l}\text { CV }- \\
\text { medium }\end{array}$ & $\begin{array}{l}\text { CV - } \\
\text { high }\end{array}$ & QC \\
ACTH $^{*}$ & $1.0 \mathrm{ng} / \mathrm{L}$ & $2,000 \mathrm{ng} / \mathrm{L}$ & $5.4 \%$ & $3.5 \%$ & $3.7 \%$ \\
Aldosterone $^{* *}$ & $25 \mathrm{ng} / \mathrm{L}$ & $1,300 \mathrm{ng} / \mathrm{L}$ & $8.2 \%$ & $7.3 \%$ & $8.8 \%$ \\
Renin** $^{* *}$ & $2.5 \mathrm{mU} / \mathrm{L}$ & $600 \mathrm{mu} / \mathrm{L}$ & $7.4 \%$ & $6.3 \%$ & $6.3 \%$ \\
\hline
\end{tabular}

*manufacturers reported performance ${ }^{* *}$ internal validation, Clinical Chemistry department, University Hospital Southampton

$\mathrm{ACTH}=$ adrenocorticotropic hormone; $\mathrm{CV}=$ coefficient of variation; $\mathrm{QC}=$ quality control
Table 2. Participants characteristics and baseline

hormone concentrations

$\begin{array}{lll}\text { ACTH, } & \text { Renin, } & \text { Aldosterone, } \\ \mathrm{n}=15 & \mathrm{n}=16 & \mathrm{n}=16\end{array}$

Mean age, years $\quad 54(19-80) \quad 52(19-71) \quad 52(19-71)$

(range)

\begin{tabular}{llll} 
Male & $53 \%$ & $44 \%$ & $44 \%$ \\
Median baseline & $18.9(7.7-$ & $16.7(3.4-$ & $72(34-339)$ \\
EDTA plasma (range) & $759.6) \mathrm{ng} / \mathrm{L}$ & $64.3) \mathrm{mU} / \mathrm{L}$ & $\mathrm{ng} / \mathrm{L}$ \\
$\begin{array}{l}\text { Median baseline } \\
\text { serum gel (range) }\end{array}$ & $19.1(8.0-$ & $19.4(3.7-$ & $72(38-343)$ \\
$\begin{array}{l}\text { p-value, serum vs } \\
\text { plasma }\end{array}$ & 0.2 & $57.1) \mathrm{mU} / \mathrm{L}$ & $\mathrm{ng} / \mathrm{L}$ \\
\hline
\end{tabular}

$\mathrm{ACTH}=$ adrenocorticotropic hormone; EDTA = Ethylenediaminetetraacetic acid

\section{Hormone stability}

Stability for all three hormones over 48 hours, by storage condition, is shown in Fig 1.

\section{ACTH}

ACTH collected into EDTA plasma was stable at room temperature for at least 6 hours (Fig 1A): mean change at 6 hours was $-2.6 \%$ (95\% CI -9.7 to 4.5). By 24 hours, levels in EDTA plasma at room temperature had dropped to $84.9 \%$ of baseline. In contrast, ACTH showed rapid decline after 1 hour in serum gel unless refrigerated: mean change (serum, room temperature) at 6 hours was $-52.6 \%(95 \% \mathrm{CI}-60.3$ to -44.9$)$. Chilling at $4^{\circ} \mathrm{C}$ did not affect ACTH stability in plasma but reduced degradation in serum (change at 6 hours in serum $-53 \%$ (95\% CI -60 to - 45) at room temperature, $-12.2 \%(95 \% \mathrm{CI}-18.2$ to -6.2$)$ at $\left.4^{\circ} \mathrm{C}\right)$.

\section{Aldosterone}

Aldosterone (Fig 1B) was stable at room temperature for at least 48 hours whether collected in serum gel (mean change at 48 hours $-0.3 \%$ ( $95 \% \mathrm{CI}-4.6$ to 4.0$)$ ) or EDTA plasma (change at 48 hours $+0.7 \%(95 \%$ CI -3.7 to 5.2$)$ ).

\section{Renin}

Renin (Fig 1C) was stable at room temperature for at least 6 hours when collected in serum gel: mean change at 6 hours was $-1.9 \%$ (95\% CI -7.0 to 3.2). Renin collected on EDTA plasma dropped by over $10 \%$ after 6 hours at room temperature (change $-10.4 \%$ (95\% CI -17.9 to -2.9$)$.

\section{Discussion}

This study demonstrates that ACTH, aldosterone and renin are stable for at least 6 hours at room temperature when collected into EDTA plasma (ACTH and aldosterone) or serum gel (aldosterone and renin). This means that complex sample handling may not be required and samples could be collected outside of a hospital setting, provided they can be processed within 6 hours. At the diagnosis of adrenal insufficiency, ACTH could be measured using the baseline Synacthen ${ }^{\mathrm{TM}}$ test sample 

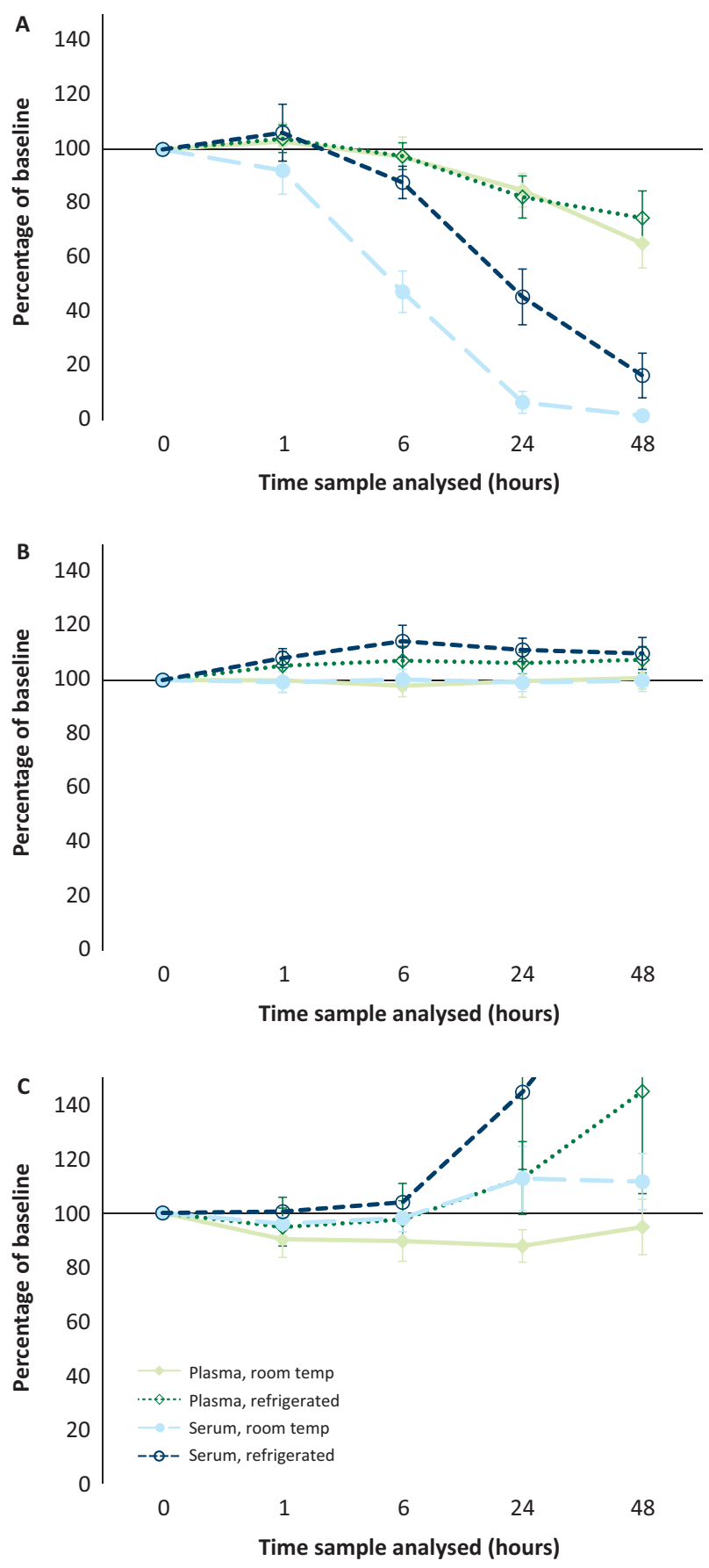

Fig 1. Degradation of biochemical assays by time after taking sample. A - adrenocorticotropic hormone (ACTH); B - aldosterone; C - renin. Error bars represent $95 \%$ confidence limits.

(even if it has not been immediately processed), facilitating availability of a pre-treatment ACTH result. A baseline Synacthen ${ }^{\text {TM }}$ test EDTA sample can be sent routinely and ACTH measured on this sample if hypoadrenalism is confirmed.

We have chosen a stability threshold of $10 \%$ for this study; this is commonly used as a threshold for acceptable preanalytic stability. ${ }^{5-7}$ Accepting small $(<10 \%)$ pre-analytic variation in these assays to facilitate sample collection would appear a reasonable trade off given the large variation already present within and between assays for these hormones. For example, intra-assay analytical variation of over $20 \%$ appears common for the measurement of ACTH with variation $<15.5 \%$ considered acceptable. ${ }^{8,9}$

Our results are consistent with recent studies using modern assays that suggest that ACTH is stable for at least 6 hours at room temperature in whole blood collected onto EDTA plasma with some studies suggesting longer stability (up to 24 hours). ${ }^{6,7,10}$ They are also consistent with previous research suggesting greater post-centrifugation stability in EDTA plasma than serum gel. ${ }^{11}$ Previous research into the stability of aldosterone is also consistent with our findings, with aldosterone previously demonstrated to be stable for over 13 hours in both EDTA plasma and serum gel. ${ }^{6}$

There is little previously published data on the stability of directly measured renin. One small study $(n=3)$ suggested stability in whole blood collected on to serum for 72 hours. ${ }^{12}$ Another study of 12 participants has suggested a decline of over $10 \%$ in EDTA plasma at 2 hours compared with immediately processed samples but did not assess stability in serum. ${ }^{13}$ Our finding of different renin concentrations in serum gel and EDTA plasma is in contrast to previous research showing similar concentrations of directly measured renin when measured in serum and plasma $(n=15) .{ }^{12}$ Dilution due to EDTA additive is reported as $<1 \%$ using this collection tube (Sarstedt, UK). The increase in renin concentration over time demonstrated in our refrigerated samples is a known phenomenon due to the conversion of prorenin to a renin-like conformation with prolonged incubation at low temperatures. ${ }^{14}$

\section{Limitations}

Limitations of our study include that we have only used a single assay type for each stability study. It is possible that stability profiles will vary between assays with different epitope binding sites, for example peripheral fragmentation of a molecule may reduce detection rates for an assay with a peripheral binding site, but may not affect an assay using a monoclonal antibody that binds more centrally. Therefore, while our results are likely to apply to other assays, this should be confirmed before changing practice if a laboratory is using a different assay. In addition, we have not assessed the effect of haemolysis, which may interfere in the measurement of plasma ACTH concentrations. ${ }^{15}$ Immediate chilling of samples and prompt analysis has been shown to improve detection of ACTH where haemolysis has occurred. ${ }^{15}$

\section{Conclusions}

Our results suggest that current laboratory requirements for ACTH and aldosterone/renin sample handling are unnecessarily restrictive. Our results support that, by using approriate preservatives, ACTH and aldosterone/renin can be measured on samples collected at room temperature and processed within 6 hours. This would facilitate clinical assessment of these analytes.

\section{Author contributions}

AJC, AGJ, TJM and BAK designed the study. AJC, AGJ and BAK collected data. AJC and AGJ analysed data with helpful discussion from BV and TJM. AGJ wrote the first draft of the manuscript, which was subsequently reviewed and edited by all authors. 


\section{Conflicts of interest}

The authors declare that they have no conflicts of interest.

\section{Funding}

This project was funded by the Royal Devon and Exeter NHS Foundation Trust NIHR Small Grant Scheme and by the Exeter NIHR Clinical Research Facility. AGJ was an NIHR doctoral research fellow and is an NIHR clinician scientist. TJM is an NIHR CSO fellow. BAK is a core staff member of the NIHR Exeter Clinical Research Facility. The views given in this paper do not necessarily represent those of NIHR, the NHS or the Department of Health.

\section{Acknowledgements}

We thank Andrew Hattersley (University of Exeter) for helpful discussion and Carol Maguire (Southampton University Hospital), Adrian Cudmore, Steven Spaull and Clinical Chemistry MTOS (Royal Devon and Exeter Hospital) for assistance with assay setup, technical aspects and/or sample handling and analysis.

\section{References}

1 Funder JW, Carey RM, Fardella C et al. Case detection, diagnosis, and treatment of patients with primary aldosteronism: an endocrine society clinical practice guideline. J Clin Endocrinol Metab 2008;93:3266-81.

2 Boscaro M, Arnaldi G. Approach to the patient with possible Cushing's syndrome. J Clin Endocrinol Metab 2009;94:3121-31.

3 AssayFinder. www.assayfinder.com [Accessed 27 October 2017].

4 McDonald TJ, Perry MH, Peake RW et al. EDTA improves stability of whole blood C-Peptide and insulin to over 24 hours at room temperature. PLoS One 2012;7:e42084.

5 Livesey JH, Ellis MJ, Evans MJ. Pre-analytical requirements. Clin Biochem Rev 2008;29(Suppl 1):S11-5.
6 Jane Ellis M, Livesey JH, Evans MJ. Hormone stability in human whole blood. Clin Biochem 2003;36:109-12.

7 Oddoze C, Lombard E, Portugal H. Stability study of 81 analytes in human whole blood, in serum and in plasma. Clin Biochem 2012;45:464-9.

8 Pecori Giraldi F, Saccani A, Cavagnini F, Study Group on the Hypothalamo-Pituitary-Adrenal Axis of the Italian Society of Endocrinology. Assessment of ACTH assay variability: a multicenter study. Eur J Endocrinol 2011;164:505-12.

9 Harris EK. Proposed goals for analytical precision and accuracy in single-point diagnostic testing. Theoretical basis and comparison with data from College of American Pathologists proficiency surveys. Arch Pathol Lab Med 1988;112:416-20.

10 Reisch N, Reincke M, Bidlingmaier M. Preanalytical stability of adrenocorticotropic hormone depends on time to centrifugation rather than temperature. Clin Chem 2007;53:358-9.

11 Evans MJ, Livesey JH, Ellis MJ et al. Effect of anticoagulants and storage temperatures on stability of plasma and serum hormones. Clin Biochem 2001;34:107-12.

12 de Bruin RA, Bouhuizen A, Diederich S et al. Validation of a new automated renin assay. Clin Chem 2004;50:2111-6.

13 Locsei Z, Racz K, Patocs A et al. Influence of sampling and storage conditions on plasma renin activity and plasma renin concentration. Clinica Chimica Acta 2009;402:203-5.

14 Deinum J, Derkx FH, Schalekamp MA. Improved immunoradiometric assay for plasma renin. Clin Chem 1999;45:847-54.

15 Livesey JH, Dolamore B. Stability of plasma adrenocorticotrophic hormone (ACTH): influence of hemolysis, rapid chilling, time, and the addition of a maleimide. Clin Biochem 2010;43:1478-80.

Address for correspondence: Dr A G Jones, NIHR Exeter Clinical Research Facility, University of Exeter Medical School, Barrack Road, Exeter EX2 5DW, UK.

Email: angus.jones@exeter.ac.uk 